ORIGINAL

\title{
Hábitos de amamantamiento del ganado bovino Chinampo (Bos taurus) de México
}

\author{
Nursing habits of Chinampo cattle (Bos taurus) from México
}

José Espinoza $\mathrm{V}_{1}^{1 *} \mathrm{Ph} . \mathrm{D}$, Rigoberto López $\mathrm{A}_{1}^{2} \mathrm{M} . \mathrm{Sc}$, Ricardo Ortega $\mathrm{P}_{1}^{1,2} \mathrm{M} . \mathrm{Sc}$, Alejandro Palacios E, ${ }^{1}$ Ph.D, Ariel Guillén T, ${ }^{1}$ Ph.D, Hugo Hernández C, ${ }^{1}$ Ph.D.

\begin{abstract}
${ }^{1}$ Universidad Autónoma de Baja California Sur. Departamento de Zootecnia. Cuerpo Académico Producción Animal Sustentable. Carretera al Sur, km. 5.5, La Paz, BCS, México. ${ }^{2}$ Centro de Investigaciones Biológicas del Noroeste, La Paz, BCS, México. *Correspondencia: jlvilla@uabcs.mx
\end{abstract}

Recibido: Mayo de 2010; Aceptado: Junio de 2011.

\section{RESUMEN}

Objetivo. Conocer los hábitos de amamantamiento de becerros Chinampos (Bos taurus) en diferentes etapas de su lactancia. Materiales y métodos. Se utilizaron 20 vacas con su cría. A partir de los 30 hasta los 180 días de la lactancia los animales fueron observados durante 24 horas continuas cada mes registrando la frecuencia con la que los becerros mamaron y la duración de cada amamantamiento. Resultados. Los becerros mamaron con mayor frecuencia entre las $06: 00$ y las $07: 00 \mathrm{hr}$, se observaron otros picos a las 10:00 y a las 19:00 hr. La frecuencia promedio de amamantamiento en 24 horas fue de 4.9 0.21 utilizando un total de $66.9 \pm 3.1$ minutos. Los becerros de 180 días de edad mamaron menos veces comparados con los de edades inferiores a los 150 días; la duración del amamantamiento disminuyó a los 180 días de edad $(p<0.05)$. Las hembras mamaron con más frecuencia que los machos $(p<0.05)$ pero el tiempo que utilizaron para esta actividad fue similar entre sexos $(p>0.05)$. Conclusiones. La frecuencia de amamantamientos es mayor durante las primeras horas de la mañana y al final de la tarde. Hasta los 5 meses de edad la frecuencia y el tiempo de amamantamiento no variaron pero se redujeron a los 180 días y las hembras mamaron con más periodicidad que los machos.

Palabras clave: Bovinos, comportamiento animal, lactación (Fuente: CAB, AIMS ) 


\section{ABSTRACT}

Objective. To determine the nursing habits of Chinampo calves (Bos taurus) during different stages of lactation. Materials and methods. Twenty cows with their calves were used. The animals were observed from day 30 to 180 of lactation during 24 continuous hours each month, recording the frequency with which the calves nursed and the duration of each nursing. A linear model was used and correlations between age, weight of calves and milk production of cows, with the frequency and duration of nursing have been established. The means were compared by Tukey test. Results. The calves nursed with higher frequency between 06:00 and 07:00; other peaks were observed at 10:00 and at 19:00 hours. The average frequency of nursing in 24 hours was $4.9 \pm 0.21$ using a total of $66.9 \pm 3.1$ minutes. The calves of 180 days of age nursed fewer times with respect to those of ages below 150 days; the duration of nursing decreased at 180 days of age $(p<0.05)$. The females nursed with more frequency than the males $(p<0.05)$, but the time they occupied for this activity was similar between males and females $(p>0.05)$. Conclusions. The frequency of nursing is higher during the first morning and late afternoon hours. Up until 5 months of age the frequency and time of nursing did not vary, but were reduced at 180 days. The females nursed with more periodicity than the males.

Key words: Animal behavior, cattle, lactation (Source: CAB, AIMS).

\section{INTRODUCCIÓN}

En la república mexicana existe una gran diversidad genética de bovinos que incluye diferentes grupos raciales adaptados a las distintas regiones agroecológicas. Sin embargo, muchas de estas poblaciones de ganado aún no han sido caracterizadas. Tal es el caso del bovino criollo Chinampo (Bos taurus) de Baja California Sur, el cuál proviene del ganado que trajeron los Jesuitas a la península de Baja California a finales del siglo XVII. Entre esos bovinos destacaron algunas variedades que al exponerse al ambiente de la región durante muchas generaciones, la selección natural produjo animales adaptados a las condiciones de aridez, conocidos localmente como Chinampos (1). Actualmente, estos animales se explotan en zonas marginadas de la entidad y durante la estación de sequía, las vacas y los toros adultos pesan $255 \pm 9$ y $345 \pm 15 \mathrm{~kg}$, respectivamente (2).

La caracterización de una raza bovina debe incluir aspectos relacionados con el comportamiento animal como los hábitos de amamantamiento de los becerros. Los hábitos de amamantamiento en bovinos han sido estudiados en razas especializadas en producción de carne $(3,4)$. Algunos trabajos han evaluado estas características en razas Bos taurus (5), Bos índicus $(6,7)$ y sus cruzamientos (8). Sin embargo, en el ganado bovino Chinampo Mexicano no se tienen antecedentes de investigación al respecto. Este ganado se explota en un sistema de producción de doble propósito. El sistema incluye la separación de los becerros de sus madres permitiendo que mamen una vez al día, esto estimula la bajada de la leche y las vacas son ordeñadas a mano; por esa razón es necesario conocer la frecuencia de amamantamiento de las crías y el tiempo requerido para ello, cuando se mantienen con su madre. En base a los planteamientos anteriores, el objetivo de este estudio fue caracterizar los hábitos de amamantamiento de becerros criollos Chinampos durante sus primeros 6 meses de vida.

\section{MATERIALES Y MÉTODOS}

Sitio de estudio. La presente investigación se realizó en la posta zootécnica de la Universidad Autónoma de Baja California Sur, en La Paz, B.C.S., México, ubicada en las coordenadas geográficas $26^{\circ} 06^{\prime} 01^{\prime \prime} \mathrm{N}$ y $110^{\circ} 0^{\prime} 0^{\prime \prime}$ O, a $33 \mathrm{msnm}$ (9). El clima predominante, según la clasificación 
de Köppen es BW (H) HW (X), siendo este clima seco y cálido con lluvias en verano, invierno y escasas todo el año; la precipitación pluvial y la temperatura media anual son de $195,4 \mathrm{~mm}$ y $28,70 \mathrm{C}$, respectivamente (9).

Animales. Se utilizaron 20 vacas Chinampas con sus crías del mismo grupo racial. Las vacas tuvieron un peso promedio de $370 \mathrm{~kg}$; todos los partos ocurrieron en un lapso de 7 días. El peso promedio de las crías al nacimiento fue de $25.0 \pm 0.3 \mathrm{~kg}$. Los animales se mantuvieron en un corral alimentándose con heno de alfalfa ( $P C=18 \%$, $\mathrm{EM}=1.9 \mathrm{Mcal} / \mathrm{kg}$ ), suplemento alimenticio $(\mathrm{PC}=25 \%, \mathrm{EM}=3.0 \mathrm{Mcal} / \mathrm{kg})$ y agua a libre acceso para cubrir sus requerimientos nutricionales. Las crías permanecieron con su madre desde el nacimiento hasta el destete, a los 180 días de edad.

Mediciones. Para determinar la frecuencia y duración del amamantamiento, los animales se observaron de manera permanente durante 24 horas a los 30, $60,90,120,150$ y 180 días de edad en promedio. En cada periodo de observación, toda vez que un becerro mamó se registró con un reloj digital la hora de inicio y de finalización. Se consideró como el inicio del amamantamiento cuando el becerro tomó la teta e inició la ingestión de leche, y la finalización cuando por voluntad propia o por rechazo de la madre dejó de mamar y no volvió a intentarlo en un lapso mayor de 30 segundos.

Las observaciones y mediciones en cada una de las edades se realizaron a partir de las 06:00 hr de un día, hasta las 06:00 hr del día siguiente. Durante la noche se dispuso de luz artificial de baja intensidad. El observador se mantuvo a una distancia de $50 \mathrm{~m}$ de los animales.

Se estimó el consumo de leche por los becerros cada mes a partir de los 30 días, hasta los 180 días de edad. Para esto se utilizó la técnica de pesaje y amamantamiento del ternero (10). Esta técnica consiste en la separación de las vacas de sus crías a las 18:00 hr, los becerros quedan en un corral y sus madres pasan a un potrero. Al día siguiente (06:00 hr) las vacas y sus crías se reúnen permitiendo el amamantamiento durante 30 minutos considerando que con esto la ubre queda vacía; se separaban otra vez los terneros de sus madres y a las 18:00 hr de ese día se pesan antes y después de un amamantamiento de 30 minutos.

La diferencia en el peso de los terneros antes y después del amamantamiento se considera la producción de leche en un periodo de $12 \mathrm{hr}$. Una vez más se hace la separación de los animales y a las 06:00 hr del día siguiente se repite el procedimiento. La suma de las diferencias de peso de los terneros en cada ocasión se consideró como la producción de leche en un lapso de 24 horas.

Las variables registradas fueron la frecuencia (número de amamantamientos) y el tiempo promedio de amamantamiento (minutos que duró cada amamantamiento) en 24 horas. La frecuencia se expresó de manera descriptiva a través de las 24 horas del día. La frecuencia y duración del amamantamiento, así como el consumo de leche por los becerros se compararon en función de la edad (30, 60, 90, 120, 150 y 180 días) y el sexo de las crías.

Análisis estadístico. Para el análisis estadístico se utilizó un modelo lineal general. Se establecieron correlaciones entre la edad, el peso de los becerros y la producción de leche de sus madres, con la frecuencia y duración del amamantamiento. Las medias se compararon mediante pruebas de Tukey. Todos los procedimientos estadísticos se realizaron a través del programa estadístico SAS (11).

\section{RESULTADOS}

El patrón general de amamantamiento de los becerros durante las 24 horas del día, desde los 30 hasta los 180 días de edad se describe en la figura 1. La mayor parte de la actividad se concentró entre las 06:00 y 07:00 hr. Otros picos de menor magnitud se presentaron a las 10:00 hr y entre las 19:00 y las 20:00 hr. 


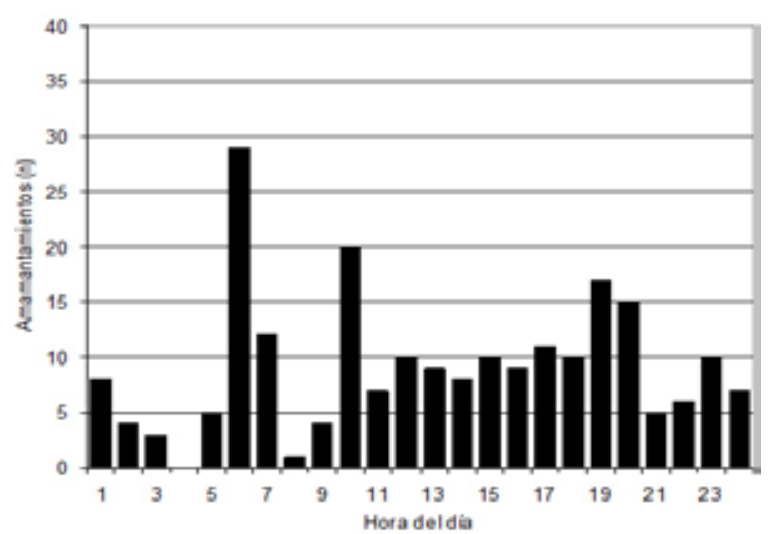

Figura 1. Distribución del total de amamantamientos en un lapso de 24 horas en becerros Chinampos.

Al analizar los datos de manera conjunta (becerros de todas las edades y de ambos sexos), la frecuencia promedio de amamantamientos en 24 horas fue de $4.9 \pm 0.21$ veces. Para ello los becerros utilizaron un total de $66.9 \pm 3.1 \mathrm{~min}$.

Cuando los datos fueron analizados en función de la edad de los becerros, se observó que la frecuencia de amamantamientos en 24 horas fue similar hasta los 150 días ( $p>0.05)$. Sin embargo, los animales de 180 días de edad mamaron menos veces $(3.3 \pm 0.39)$ que los de 120 días y los más jóvenes $(p<0.05)$.

La producción de leche promedio durante los primeros 180 días de lactación fue de $5.7 \pm 0.8 \mathrm{~kg} /$ día. Como se aprecia en la tabla 1, el peso de los becerros entre los 30 y 180 días de edad varió entre $46 \pm 8$ y $144 \pm 11 \mathrm{~kg}$ (media \pm D.E.). El tiempo que los becerros mamaron en un periodo de 24 horas fue similar en animales de diferentes edades hasta los 150 días $(p>0.05)$, sin embargo, el tiempo de amamantamiento

Tabla 1. Frecuencia y tiempo de amamantamiento (media \pm D.E.), por edad de los becerros Chinampos.

\begin{tabular}{cccc}
\hline $\begin{array}{c}\text { Edad } \\
\text { (días) }\end{array}$ & $\begin{array}{c}\text { Peso } \\
(\mathbf{k g})\end{array}$ & $\begin{array}{c}\text { Frecuencia de } \\
\text { amamantamiento }{ }^{1}\end{array}$ & $\begin{array}{c}\text { Tiempo de } \\
\text { amamantamiento }{ }^{2}\end{array}$ \\
\hline 30 & $46 \pm 8$ & $5.6 \pm 0.5^{\mathrm{a}}$ & $81.5 \pm 8.4^{\mathrm{a}}$ \\
60 & $65 \pm 11$ & $5.2 \pm 0.6^{\mathrm{a}}$ & $65.5 \pm 6.0^{\mathrm{a}}$ \\
90 & $81 \pm 16$ & $5.7 \pm 0.3^{\mathrm{a}}$ & $76.4 \pm 6.8^{\mathrm{a}}$ \\
120 & $106 \pm 24$ & $5.6 \pm 0.4^{\mathrm{a}}$ & $73.0 \pm 5.4^{\mathrm{a}}$ \\
150 & $131 \pm 31$ & $4.1 \pm 0.2^{\mathrm{ab}}$ & $64.8 \pm 7.9^{\mathrm{a}}$ \\
180 & $144 \pm 34$ & $3.3 \pm 0.3^{\mathrm{b}}$ & $40.6 \pm 4.8^{\mathrm{b}}$ \\
\hline
\end{tabular}

${ }^{1}$ Número de amamantamientos por día. ${ }^{2}$ Minutos que amamantaron en 24 horas. ${ }^{a b}$ Literales diferentes en las columnas indican desigualdad $(p<0.05)$. a los 180 días fue menor $(p<0.05)$. Las correlaciones de la edad de los becerros con la frecuencia y duración del amamantamiento fueron de -0.57 y -0.55 , respectivamente $(p<0.05)$. De manera similar, el peso de las crías tuvo una correlación negativa $(p<0.05)$ con la frecuencia $(-0.51)$ y con la duración $(-0.45)$ del amamantamiento.

En la figura 2 se observa que la frecuencia de amamantamientos fue mayor $(p<0.05)$ en las hembras $(5.4 \pm 0.28)$ que en los machos $(4.2 \pm 0.27)$.

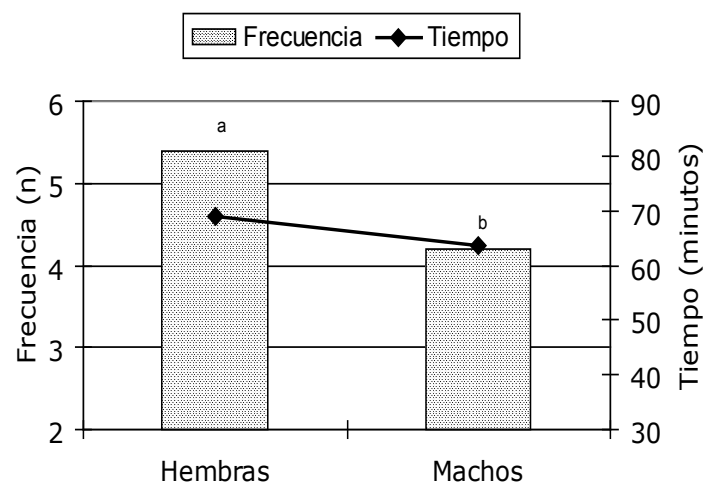

Figura 2. Frecuencia y tiempo de amamantamiento en un lapso de 24 horas en becerros Chinampos clasificados por sexo ( $a, b$ en las barras $=(p<0.05)$.

El tiempo de amamantamiento fue similar $(p>0.05)$ entre sexos $(69 \pm 4.1$ y 63.7 \pm 4.8 minutos para hembras y machos, respectivamente).

\section{DISCUSIÓN}

Los hábitos de amamantamiento registrados en este trabajo son similares a los observados en un estudio realizado con ganado Hereford y Simmental (12). Estudios similares realizados por Reindhardt y Reindhardt (7) encontraron resultados parecidos en ganado Bos índicus de Kenya, con dos momentos de máxima actividad de amamantamiento, una antes de la salida y otra antes de la puesta de sol. También, coincide con el presente trabajo el hecho de que esos autores encontraron una menor actividad de amamantamiento en horas de oscuridad. 
En otro estudio se observó que los becerros Brahman maman 2.4 veces más durante el día que en la noche (13). Consistente también con el presente estudio es el hecho de que los autores anteriores encontraron una menor actividad de amamantamiento en horas de oscuridad y que la mayor actividad nocturna se realizó en la primera mitad de la noche.

En contraste, Somerville y Lowman (4) señalan que la frecuencia de amamantamientos en becerros para carne en su primer mes de vida fue similar durante el día y la noche, pero a edades más avanzadas hubo una mayor actividad nocturna ya que los animales estaban confinados en corrales iluminados $y$ techados. En uno de los primeros estudios reportados en esta temática (3) encontraron que la frecuencia de amamantamiento en ganado Angus fue mínima durante la noche cuando el ganado no fue molestado, mientras que otros (5) observaron que becerros Bos taurus en California mamaron a toda hora, con una actividad máxima entre las 05:00 y 06:00 hr.

Una frecuencia similar (5 amamantamientos) pero con un tiempo menor (46 minutos) fueron reportados en becerros Bos taurus para carne (12). Por su parte Plasse et al (13), estudiaron las mismas variables en ganado Brahman y observaron valores inferiores a los del presente estudio (4.4 amamantamientos en 24 horas). Reinhardt y Reinhardt (7) observaron 28 becerros Bos índicus; el número promedio de amamantamientos por día durante las primeras 49 semanas de vida fue de 4.8 con una duración total de 38 minutos.

Una frecuencia de amamantamiento mayor que la de este trabajo fue determinada por Hutchison et al (6); observaron cinco becerros Bos índicus con sus madres durante 75 horas, constatando que mamaron 6.7 veces y para esto utilizaron $60.3 \mathrm{~min}$.

Resultados similares a los observados hasta los 5 meses de edad fueron reportados en ganado Hereford (12) y Charolais (4). En otros trabajos se observó una disminución de la frecuencia de amamantamiento conforme avanzó la edad de las crías (7, 14). En un estudio de Drewry et al (3) los becerros Angus mamaron 4.6, 4.8 y 3.0 a los 30, 90 y 180 días de edad, respectivamente. Las discrepancias con el presente estudio pueden ser explicadas por el hecho de que los trabajos citados fueron realizados en condiciones de pradera o agostadero y los becerros tuvieron acceso permanente al pasto, en cambio en esta investigación los animales se mantuvieron en corral y el consumo de alimento no fue de manera deliberada; de esta forma, los becerros pudieron haber requerido de un mayor aporte de leche de sus madres. Lo anterior fue sugerido por Ansotegui et al (15) en becerros de raza para carne, al estimar una correlación negativa $(-0.62)$ entre el consumo de forraje y el consumo de leche. En otro estudio se demostró que la reducción en el consumo de leche tuvo un efecto lineal sobre el consumo de forraje (16).

De acuerdo con los resultados de este trabajo se concluye que los hábitos de amamantamiento de becerros Chinampos siguen un patrón similar al de las diferentes razas productoras de carne que reporta la literatura. La frecuencia de amamantamiento es mayor durante las primeras horas de la mañana y al final de la tarde. Hasta los 5 meses de edad la frecuencia y el tiempo de amamantamiento no variaron pero se redujeron a los 180 días y las hembras mamaron con más periodicidad que los machos. 


\section{REFERENCIAS}

1. Martínez-Balboa A. La ganadería en Baja California Sur, La Paz BCS. México: Ed JB; 1981.

2. Espinoza JL, Guevara A, Palacios A. Caracterización morfométrica y faneróptica del bovino Criollo Chinampo de México. Arch Zootec 2009; 58:277-279.

3. Drewry $\mathrm{KJ}$, Brown $\mathrm{CJ}$, Hones RS. Relationships among factors associated with mothering ability in beef cattle. J Anim Sci 1959; 18:938-946.

4. Somerville E, Lowman BG. Observations of the nursing behavior of beef cows suckling charolais cross calves. Appl Anim Ethology 1979; 5: 369-373.

5. Wagnon KA. Behavior of beef cows on a California range. California Agric Exp Sta Bull 1963.

6. Hutchison HG, Woof R, Mabon RM, Salehe JM, Rubb A. Study of the habits of Zebu cattle in Tanganyika. J Agric Sci 1962; 59:01-317.

7. Reinhardt $V$, Reinhardt A. Natural suckling performance and age of weaning in Zebu cattle (Bos indicus). J Agric Sci 1981; 96:309-312.

8. Cartwright TC, Carpenter JA. Effect of nursing habits on calf weights. J Anim Sci 1961; 20(Abstr):904.

9. Espinoza $\mathrm{J}$, López $\mathrm{R}$, Palacios $\mathrm{A}$, Ortega R, Ávila N, Murillo B. Efecto del toro sobre el comportamiento estral de vacas Chinampas (Bos Taurus) en una region tropical seca. Zootecnia Trop 2007; 25:19-28.
10. Espinoza JL, Ríos JG. Producción de leche de vacas Hereford, Angus y su cruza bajo condiciones de agostadero. Rev Mex Prod Anim 1985; 17:3-11.

11. SAS. SAS/STAT User's Guide (Release 6, 4th Ed.). Cary NC: SAS. Inst. Inc., 1990.

12. Odde KG, Kiracofe $G H$, Schalles RR. Suckling behavior in range beef calves. J Anim Sci 1985; 61:307-309.

13. Plasse D, Beltrán J, Verde 0. Frecuencia y duración de mamado en becerros Brahman. Zootecnia Trop 1984; 21:106-125.

14. Ewbank R. The frequency and the duration of the nursing periods in single suckled Hereford beef cows. Brit Vet J 1969; 2: 9-10.

15. Ansotegui RP, Havstad KM, Wallace JD, Hallford DM. Effects of milk intake on forage intake and performance of suckling range calves. J Anim Sci 1991; 69: 899-904.

16. Broesder JT, Judkins MB, Krysl LJ, Gunter SA, Barton RK. Forage intake, digestibility and digesta kinetics in calves receiving 30 or $60 \%$ milk reduction and ad libitum hay. J Anim Sci 1989; 67(Suppl 2):208. 